1.15-1.68)). This association was further strengthened in relation to HDP and SGA (Adjusted OR=1.60; (95\% CI: 1.062.29).

Conclusion This study suggests that HDP exposure may increase the risk of asthma in the offspring and the association was independent of several potential confounders. Considering that the association was larger in relation to $\mathrm{HDP} /$ SGA compared to HDP alone, placental pathology may be a common factor increasing the risk of asthma.

\section{OP100 ASSOCIATION BETWEEN HYPERTENSIVE DISORDERS OF PREGNANCY AND ATTENTION DEFICIT HYPERACTIVITY DISORDER: A POPULATION-BASED AND SIBLING- MATCHED COHORT STUDY}

\begin{abstract}
${ }^{1,2} \mathrm{GM}$ Maher* ${ }^{3}{ }^{3} \mathrm{C}$ Dalman, ${ }^{2} \mathrm{PM}$ Kearney, ${ }^{1,4} \mathrm{GW}$ O'Keeffe, ${ }^{1} \mathrm{FP}$ McCarthy, ${ }^{5} \mathrm{LC}$ Kenny, ${ }^{1,2}$ AS Khashan. ${ }^{1}$ The Irish Centre for Fetal and Neonatal Translational Research (INFANT), Cork University Maternity Hospital and University College Cork, Cork, Ireland; ${ }^{2}$ School of Public Health, University College Cork, Cork, Ireland; ${ }^{3}$ Department of Public Health Sciences, Karolinska Institutet, Stockholm, Sweden; ${ }^{4}$ Department of Anatomy and Neuroscience, University College Cork, Cork, Ireland; ${ }^{5}$ Department of Women's and Children's Health, University of Liverpool, Liverpool, UK
\end{abstract}

\subsection{6/jech-2019-SSMabstracts. 103}

Background Hypertensive disorders of pregnancy (HDP) have been linked to adverse neurodevelopmental outcomes, including attention deficit hyperactivity disorder (ADHD). However, limitations of the literature such as residual confounding, and confounding due to shared genetics need to be addressed before more definitive conclusions can be reached. Therefore, the objective of this study was to examine the association between HDP and ADHD, using large Swedish-based registry data, allowing us to control for a wide range of confounding factors including confounding due to shared genetics.

Methods All singleton live births in Sweden from 1990 to 2010 were included in the study. Data on HDP were obtained from the Medical Birth Register, and included three exposures of interest: 1. Preeclampsia 2. Chronic hypertension (both of which were classified according to ICD-9 and ICD-10 coding) 3. Preeclampsia and small for gestational age (SGA) combined, used as a proxy for the recent inclusion of uteroplacental dysfunction in the definition of preeclampsia. Data on ADHD was obtained from the National Patient Register and Prescribed Drug Register. A diagnosis of ADHD was determined in one of two ways: 1. If a diagnosis of ADHD was present in the National Patient Register, using ICD-10 coding. 2. If an individual was in receipt of ADHD medication, classified according to Anatomical Therapeutic Chemical classification system. Multivariate Cox proportional hazards regression analysis was conducted, adjusting for several perinatal and sociodemographic factors, selected a priori from previous literature. Sibling-matched analysis further controlled for shared genetic and familial confounding when an association was present in the adjusted Cox model.

Results The cohort consisted of 2,047,619 children, with $114,934(5.6 \%)$ cases of ADHD. In the adjusted Cox model, preeclampsia was associated with a $15 \%$ increase in the likelihood of ADHD (Hazard Ratio (HR): 1.15, 95\% CI: $1.12,1.19)$ compared to those unexposed to preeclampsia. Result of the sibling-matched analysis did not change materially (HR: 1.13, 95\% CI: 1.05, 1.22). The HR for preeclampsia and those born SGA was 1.43 (95\% CI: 1.31, 1.55) in the adjusted model, and 1.55 (95\% CI: 1.28, 1.88) in the sibling-matched analysis. The HR for chronic hypertensionADHD was 1.07 (95\% CI: 0.97, 1.18) in the adjusted Cox model.

Conclusion Exposure to preeclampsia or preeclampsia/SGA (i.e. SGA baby exposed to preeclampsia) was associated with ADHD, while chronic hypertension was not associated with ADHD. Placental pathology may be a common factor increasing the likelihood of ADHD given the stronger association with preeclampsia/SGA than preeclampsia alone.

\section{OP101 THE IMPACT OF CAESAREAN SECTION ON BREASTFEEDING INDICATORS IN SUB-SAHARAN AFRICA: A META-ANALYSIS OF DEMOGRAPHIC AND HEALTH SURVEYS}

${ }^{1} \mathrm{E}$ Yisma*, ${ }^{2} \mathrm{~B}$ Mol, ${ }^{1} \mathrm{~J}$ Lynch, 'L Smithers. 'School of Public Health, The University of Adelaide, Adelaide, Australia; ${ }^{2}$ Department of Obstetrics and Gynaecology, Monash University, Melbourne, Australia

\subsection{6/jech-2019-SSMabstracts. 104}

Background The association between caesarean section and breastfeeding is poorly understood in sub-Saharan Africa. We aimed to examine the impact of caesarean section on breastfeeding indicators - early initiation of breastfeeding, exclusive breastfeeding, and ever breastfeeding - in sub-Saharan Africa.

Methods We used the most recent data from 32 Demographic and Health Surveys (DHS) completed in sub-Saharan Africa. We analysed the data to examine the impact of caesarean section on breastfeeding indicators using log-Poisson regression models for each country adjusted for potential confounders. For each breastfeeding indicator, the within-country adjusted prevalence ratios were pooled in random effects meta-analysis. Results The within-country adjusted analyses showed, compared with vaginal birth, caesarean section was associated with adjusted prevalence ratios (aPR) for early initiation of breastfeeding that ranged from $0.23(95 \% \mathrm{CI}, 0 \cdot 16,0 \cdot 31)$ in Tanzania to $0.81(95 \% \mathrm{CI}, 0.64,1.02)$ in Cameroon. Similarly, the aPR for exclusive breastfeeding ranged from 0.57 (95\%CI; $0.33,0.99)$ in Senegal to $1.60(95 \% \mathrm{CI} ; 1.07,2.39)$ in Mali, while the aPR for ever breastfeeding ranged from 0.90 (95\% CI, $0.82,0.99)$ in Liberia to $1.02(95 \% \mathrm{CI}, 0.98,1.06)$ in Guinea. Meta-analysis combining the adjusted effects from 32 countries showed that caesarean section was associated with a $47 \%$ lower prevalence of early initiation of breastfeeding (pooled PR, 0.53 (95\% CI, 0.48, 0.58)), but not with exclusive breastfeeding (pooled PR, 0.93 (95\%CI; 0.86, 0.99)) nor ever breastfeeding (pooled PR, 0.98 (95\%CI; 0.98, 0.99)).

Conclusion Caesarean section had a negative influence on early initiation of breastfeeding, but showed little difference in exclusive- and ever-breastfeeding between infants born by caesarean versus vaginal birth in sub-Saharan Africa. 